\title{
Some Theorems about Formal Functions
}

\author{
By \\ Gerd Faltings*
}

\section{§o. Introduction}

It is well-known that in algebraic geometry there are very few nontrivial problems about continuation of objects from open subsets, which is a big difference to analytic geometry. So we may expect that in the algebraic version of analytic geometry, formal geometry, there are some interesting problems of this kind, and this assumption is supported by the following paper.

In there we take a local ring which is complete in some $\mathscr{I}$-adic topology and consider the formal scheme obtained by completing the spectrum of our local ring along the closed subscheme defined by $\mathscr{I}$. As all interesting formal objects on this formal scheme stem from objects on the usual spectrum of our local ring, we remove a closed subset. After that there may exist nonalgebraic formal objects, and such objects are characterized by the fact that there is no continuation of them to the whole formal scheme.

The objects we are interested in are formal meromorphic functions and formal subsheaves of algebraic formal sheaves. As it is the case in analytic geometry these two cases are connected, and any result about one of them implies a corresponding result about the other.

As application of our theory we prove certain results about connectedness, including a generalization of Zariski's connectedness-theorem.

I express my gratitude to Professor Hironaka for some very stimulating discussion, and to the Deutsche Forschungsgemeinschaft for support during the last year.

Communicated by H. Hironaka, October 19, 1978. Revised October 9, 1979.

* Mathematisches Institut, Universität Münster, Roxeler Str. 64, 4400 Münster, West Germany. 


\section{§1. Preliminaries}

In this paper all rings are supposed to be commutative with a unit, noetherian and to have a dualizing complex. The last assumption carries over to localizations and completions and implies that the formal fibers are gorenstein (compare [F]). Suppose $A$ is such a ring, which in addition is local, and $\mathscr{I} \subset \mathfrak{a}$ $\varsubsetneqq A$ two ideals. Then we denote $\operatorname{Spec}(A)$ by $X$, the closed subscheme $\operatorname{Spec}(A / \mathscr{I})$ of $X$ by $Y$, and $\operatorname{Spec}(A / \mathfrak{a}) \subseteq Y$ by $Z$. If $A$ is complete in the $\mathscr{I}$-adic topology, then $\hat{X}=\operatorname{Spf}(A)$ is the formal completion of $X$ along $Y$, and $\hat{U} \subset \hat{X}$ is its open subscheme obtained by removing $Z$. If somewhere in this paper we introduce $A, \mathscr{I}, \mathfrak{a}$ as above these notations are in force automatically, and if furthermore there occur $A_{1}, A_{2}, \ldots, \mathscr{I}_{1}, \mathscr{I}_{2}, \ldots$, and $\mathfrak{a}_{1}, \mathfrak{a}_{2}, \ldots$, the objects denoted by $X_{1}$, $X_{2}, \ldots, Y_{1}, Y_{2}, \ldots$, etc. are exactly what the reader expects them to be. $\hat{U}$ is covered by the affine open subschemes $\operatorname{Spf}\left(A_{(f)}\right)$, where $f$ moves in a system of generators of $\mathfrak{a}$ or only $\mathfrak{a} / \mathscr{I}$, and $A_{(f)}$ denotes the $\mathscr{I}$-adic completion of the usual localization $A_{f}$.

If $K^{*}$ is a dualizing complex for $A$, then it is seen as in [F] that $K^{*} \otimes_{A} A_{(f)}$ is a dualizing complex for $A_{(f)}$, and that for $f, g \in \mathfrak{a}$ the fibers of the mapping $\operatorname{Spec}\left(A_{(f g)}\right) \rightarrow \operatorname{Spec}\left(A_{(f)}\right)$ are gorenstein. If $d$ is the codimension-function of $K^{*}$ and if we denote the codimension-functions of $K^{*} \otimes_{A} A_{(f)}$ and $K^{*} \otimes_{A} A_{(f g)}$ by the same name, then for all primeideals $\mathfrak{p} \subset A d$ takes the value $d(\mathfrak{p})$ on all minimal prime overideals of $\mathfrak{p} \cdot A_{(f)}$, which are by the way all the associated prime ideals of $A_{(f)} / \mathfrak{p} \cdot A_{(f)}$. A similar conclusion holds for the basechange from $A_{(f)}$ to $A_{(f g)}$, and this allows to handle the following difficulty:

If the finitely generated $A_{(f)}$-module $M$ has only one associated prime ideal, this need not be the case for $M \otimes_{A_{(f)}} A_{(f g)}$. But $d$ takes the same value on all associated prime ideals of $M \otimes_{A_{(f)}} A_{(f g)}$.

Hence, although the primary decompositions of a coherent sheaf on $\hat{U}$ on the affine parts $\operatorname{Spf}\left(A_{(f)}\right)$ need not glue together to a global decomposition, we may get a global decomposition of we take the intersection of the primary components belonging to a fixed value of $d$. As $d$ changes with $K^{*}$, we change the numeration in the following way:

As $f$ is no unit (since $f \in \mathfrak{a} \neq A$ ), and as maximal ideals of $A_{(f)}$ contain $\mathscr{I} \cdot A_{(f)}$, they can be identified with maximal ideals of $A_{(f)} / \mathscr{I} \cdot A_{(f)} \cong(A / \mathscr{I})_{f}$ or with prime ideals $\mathfrak{q}$ of $A$ for which $(A / \mathfrak{q})_{f}$ is a field and hence $\operatorname{dim}(A / \mathfrak{q})=1$. If $\mathfrak{m}$ is 
the maximal ideal of $A$, we therefore obtain $d(\mathfrak{q})=d(\mathfrak{m})-1$, and for any prime ideal $\mathfrak{p} \subseteq A_{(f)}$ we have $\operatorname{dim}\left(A_{(f)} / \mathfrak{p}\right)=d(\mathfrak{m})-d(\mathfrak{p})-1$. So, instead of the $d \cdot$ function we can use the dimension for our parametrisation, and we obtain the following

Lemma 1. If $\mathscr{F}$ is a coherent sheaf on $\hat{U}$, there exist uniquely determined coherent subsheaves $\mathscr{G}_{i}(0 \leqq i<\operatorname{dim}(A))$ of $\mathscr{F}$, such that $\cap \mathscr{G}_{i}=(0)$ and such that for all $f \in \mathfrak{a}$ and all associated prime ideals $p$ of $\Gamma\left(\operatorname{Spf}\left(A_{(f)}, \mathscr{F} / \mathscr{G}_{i}\right)\right.$ $\operatorname{dim}\left(A_{(f)} / \mathfrak{p}\right)=i$.

We denote the sheaf of formal meromorphic functions on $\hat{U}$, which by definition is the total sheaf of fractions of $\mathcal{O}_{0}$, by $\mathscr{M}_{0}$, and its global sections by $M(\hat{U})$. The sections of $\mathscr{M}_{0}$ over some $\operatorname{Spf}\left(A_{(f)}\right)$ can be identified with the total ring of fractions of $A_{(f)}$, and the intersection of the support of a global formal meromorphic function with $\operatorname{Spf}\left(A_{(f)}\right)$ is the union of certain primary components of $\operatorname{Spf}\left(A_{(f)}\right)$. If the local rings of $\hat{U}$ are integral domains, then the support of a formal meromorphic function is open and closed, and hence $M(\hat{U})$ is a field if and only if $\hat{U}$ (or $Y-Z$ ) is connected.

For an integral domain $A$ we denote by $Q(A)$ its field of fractions. An extension of fields $K \subseteq L$ is called regular if it is separable and if $K$ is algebraically closed in $L$. This is equivalent to the fact that for some algebraically closed extension of $K, \bar{K}, L \otimes_{K} \bar{K}$ is an integral domain.

We have the following result:

Lemma 2. If $K \subseteq L$ is a regular extension of fields, then $K\left(\left(T_{1}, \ldots, T_{n}\right)\right)$ $\subseteq L\left(\left(T_{1}, \ldots, T_{n}\right)\right)$ is regular too.

Proof. We first reduce to the case of an algebraically closed $K$. If $\bar{K}$ is an algebraically closed extension of $K$, we claim that the natural mapping

$$
L\left(\left(T_{1}, \ldots, T_{n}\right)\right) \otimes_{K\left(\left(T_{1}, \ldots, T_{n}\right)\right)} \bar{K}\left(\left(T_{1}, \ldots, T_{n}\right)\right) \longrightarrow Q\left(L \otimes_{K} \bar{K}\right)\left(\left(T_{1}, \ldots, T_{n}\right)\right)
$$

is an injection.

If this were not true we could find $x_{i} \in L\left(\left(T_{1}, \ldots, T_{n}\right)\right)$ and $y_{i} \in \bar{K}\left(\left(T_{1}, \ldots, T_{n}\right)\right)$ for $1 \leqq i \leqq m$, such that $x_{i} \neq 0$ for all $i$ and such that the $y_{i}$ are linearly independent over $K\left(\left(T_{1}, \ldots, T_{n}\right)\right)$, so that $\sum_{i=1}^{m} x_{i} \cdot y_{i}=0$ in $Q\left(L \otimes_{K} \bar{K}\right)\left(\left(T_{1}, \ldots, T_{n}\right)\right)$. After multiplication with nonzero factors from $L\left[\left[T_{1}, \ldots, T_{n}\right]\right]$ resp. $\bar{K}\left[\left[T_{1}, \ldots, T_{n}\right]\right]$ we may assume that $x_{i} \in L\left[\left[T_{1}, \ldots, T_{n}\right]\right]$ and $y_{i} \in \bar{K}\left[\left[T_{1}, \ldots, T_{n}\right]\right]$.

If $s: L \rightarrow K$ is a $K$-linear retraction of the injection $K \hookrightarrow L$, we can extend $s$ to a retraction $L\left[\left[T_{1}, \ldots, T_{n}\right]\right] \rightarrow K\left[\left[T_{1}, \ldots, T_{n}\right]\right]$ by letting $s$ act on the coef- 
ficients of the powerseries, and as $s$ induces a retraction $L \otimes_{K} \bar{K} \rightarrow \bar{K}$ of the injection $\bar{K} \hookrightarrow L \otimes_{K} \bar{K}$, we obtain in the same way a $\bar{K}\left[\left[T_{1}, \ldots, T_{n}\right]\right]$-linear retraction $s:\left(L \otimes_{K} \bar{K}\right)\left[\left[T_{1}, \ldots, T_{n}\right]\right] \rightarrow \bar{K}\left[\left[T_{1}, \ldots, T_{n}\right]\right]$. So we obtain that

$$
0=s\left(\sum_{i=1}^{m} x_{i} \cdot y_{i}\right)=\sum_{i=1}^{m} s\left(x_{i}\right) \cdot y_{i} \text {. }
$$

As $s\left(x_{i}\right) \in K\left[\left[T_{1}, \ldots, T_{n}\right]\right]$ and as the $y_{i}$ are linearly independent over this ring, $s\left(x_{i}\right)=0$ for all $i$. But if $x_{i} \neq 0$, we can clearly find an $s$ with $s\left(x_{i}\right) \neq 0$. This proves our claim, which obviously allows us to assume that $K$ is algebraically closed.

In this case the separability of $K\left(\left(T_{1}, \ldots, T_{n}\right)\right) \subseteq L\left(\left(T_{1}, \ldots, T_{n}\right)\right)$ is clear, since the $T_{i}$ form a $p$-base of $K\left(\left(T_{1}, \ldots, T_{n}\right)\right)$ and are part of a $p$-base of $L\left(\left(T_{1}, \ldots, T_{n}\right)\right)$ (if $\operatorname{char}(K)=p>0$ ).

Hence it remains to prove that $K\left(\left(T_{1}, \ldots, T_{n}\right)\right)$ is algebraically closed in $L\left(\left(T_{1}, \ldots, T_{n}\right)\right)$, which is equivalent to the fact that $K\left[\left[T_{1}, \ldots, T_{n}\right]\right]$ is integrally closed in $L\left[\left[T_{1}, \ldots, T_{n}\right]\right]$.

We first consider the case $n=1$ : Suppose $E \subseteq L((T))$ is a field containing and being finite over $K((T))$. Then the integral closure of $K[[T]]$ in $E$ is a discrete valuation-ring, and the corresponding valuation of $E$ is the unique extension of the $T$-adic valuation on $K((T))$ and therefore it is equal to the restriction of the $T$-adic valuation on $L((T))$ to $E$. This implies that the ramification index of $K((T)) \subseteq E$ is one, which of course also is the case for the degree of the extension of the residue-fields, since $K$ is algebraically closed. As the product of these two quantities is the degree of the extension $K((T)) \subseteq E$, $E$ must be equal to $K((T))$. For general $n$ we take a powerseries $f\left(T_{1}, \ldots, T_{n}\right)$ $\in L\left[\left[T_{1}, \ldots, T_{n}\right]\right]$, which is integral over $K\left[\left[T_{1}, \ldots, T_{n}\right]\right]$. For arbitrary elements $x_{1}, \ldots, x_{n} \in K$ this implies that $f\left(x_{1} \cdot S, \ldots, x_{n} \cdot S\right) \in L[[S]]$ is integral over $K[[S]]$ and hence an element of $K[[S]]$ by the previous considerations. As $K$ is infinite, this implies immediately $f \in K\left[\left[T_{1}, \ldots, T_{n}\right]\right]$. This proves our lemma.

We denote by $\hat{\otimes}$ the complete tensorproduct as in $[\mathrm{S}]$, Chapter V, 2.

Lemma 3. Suppose $K$ is a field, $\mathfrak{p}$ and $\mathfrak{q} \subset K\left[\left[T_{1}, \ldots, T_{n}\right]\right]$ prime ideals, such that $Q(A / \mathfrak{p})$ is a regular extension of $K$. Then $(A / \mathfrak{p}) \hat{\otimes}_{K}(A / \mathfrak{q})$ is an integral domain, and its field of fractions is a regular extension of $Q(A / \mathfrak{q})$.

Proof. If $X_{1}, \ldots, X_{m}$ is a system of parameters for $A / \mathfrak{q}$, then $A / \mathfrak{q}$ is finite over $K\left[\left[X_{1}, \ldots, X_{m}\right]\right]$, and $(A / \mathfrak{p}) \widehat{\otimes}_{K}(A / \mathfrak{q})$ is isomorphic to 
$(A / \mathfrak{p})\left[\left[X_{1}, \ldots, X_{m}\right]\right] \otimes_{K\left[\left[X_{1}, \ldots, X_{m}\right]\right]}(A / \mathfrak{q})$ and hence contained in $(A / \mathfrak{p})\left[\left[X_{1}, \ldots, X_{m}\right]\right]$ $\otimes_{K\left[\left[x_{1}, \ldots, X_{m}\right]\right]} Q(A / \mathfrak{q})$.

Since $K\left(\left(X_{1}, \ldots, X_{m}\right)\right) \subseteq Q\left((A / \mathfrak{p})\left[\left[X_{1}, \ldots, X_{m}\right]\right]\right) \subseteq Q(A)\left(\left(X_{1}, \ldots, X_{m}\right)\right)$, the first extension is regular by Lemma 2 and our claim can be derived by standard field-theory.

Elementary facts from commutative algebra, as they are for example listed in $[M]$, are used without explicit reference.

\section{§2. G3-Theorems}

Theorem 1. Suppose $A$ is a complete local integral domain containing a field, $\mathscr{I} \subset A$ an ideal, which can be generated by $n$ elements for some natural number $n$ and for which $\operatorname{dim}(A / \mathscr{I})=\operatorname{dim}(A)-n$. If $\mathfrak{a} \supset \mathscr{I}$ is another ideal with $\operatorname{dim}(A / \mathfrak{a}) \leqq \operatorname{dim}(A / \mathscr{I})-2$, then $\hat{U}$ is $G 3$, or equivalently, $M(\hat{U})=Q(A)$ ( $\hat{U}$ as in the introduction).

Proof. If $x_{1}, \ldots, x_{n}$ is a system of generators of $\mathscr{I}$, we can find a complete regular local subring $R \subseteq A$, such that $A$ is finite over $R\left[\left[x_{1}, \ldots, x_{n}\right]\right]$ and such that $\operatorname{dim}(R)=\operatorname{dim}(A / \mathscr{I})$. We further may replace $\mathfrak{a}$ by any smaller ideal fulfilling the same assumptions, since this means removing some part of $\hat{U}$ and since the support of a nonzero formal meromorphic function cannot be contained in the removed part:

For on some $\operatorname{Spf}\left(A_{(f)}\right)$ with $f \in \mathfrak{a}$ our function can be identified with an element of the total ring of fractions of $A_{(f)}$. As $A$ is quotient of a regular local ring, the support of this element has pure dimension $\operatorname{dim}\left(A_{(f)}\right)=\operatorname{dim}(A)-1$, and hence its intersection with $V(\mathscr{I})$, which is the support of our formal meromorphic function on $\operatorname{Spf}\left(A_{(f)}\right)$, is either void or of dimension $\geqq \operatorname{dim}(A)-1-n$ $>\operatorname{dim}(A / \mathfrak{a})$.

If we define $A_{1} \subseteq A$ as $A_{1}=R\left[\left[x_{1}, \ldots, x_{n}\right]\right], \mathscr{I}_{1}=\left(x_{1}, \ldots, x_{n}\right) \cdot A_{1}, \mathfrak{a}_{1}=\mathfrak{a} \cap A_{1}$, we may replace a by $\mathfrak{a}_{1} \cdot A$. Then $\hat{U}$ is finite over $\hat{U}_{1}$, and it is immediate that $M(\hat{U})=M\left(\hat{U}_{1}\right) \otimes_{Q\left(A_{1}\right)} Q(A)$.

Hence it is enough to prove our theorem for $\hat{U}_{1}$. If $f \in M\left(\hat{U}_{1}\right)$ is a formal meromorphic function, the ideal $\mathscr{I}=\mathcal{O}_{O_{1}} \cap f^{-1} \cdot \mathcal{O}_{\tilde{U}_{1}} \subseteq \mathcal{O}_{O_{1}}$ defines the polar-divisor of $f . \mathscr{I}$ is locally free, and so is $\mathscr{L}=\mathscr{H}_{a m_{O_{U_{1}}}}\left(\mathscr{I}, \mathcal{O}_{O_{1}}\right) . f$ and the constant function 1 define global sections of $\mathscr{L}$, and we have to prove that these sections are linearly dependent over $A_{1}$. Hence it remains to prove the following claim: 
If $\mathscr{L}$ is a formal line-bundle on $\hat{U}_{1}$, then $\Gamma\left(\hat{U}_{1}, \mathscr{L}\right)$ is a finitely generated, torsion-free $A_{1}$-module of rank $\leqq 1$.

We prove this claim by induction over $n$, where $A_{1}=R\left[\left[x_{1}, \ldots, x_{n}\right]\right]$. If $n=0$, then $\mathscr{L} \cong \mathcal{O}_{\mathbb{O}_{1}}$, and $\Gamma\left(\hat{U}_{1}, \mathscr{L}\right) \cong A_{1}$, since $\operatorname{dim}\left(A_{1} / \mathfrak{a}_{1}\right) \leqq \operatorname{dim}\left(A_{1}\right)-2$.

If $n>0$, we have an exact sequence

$$
0 \longrightarrow \Gamma\left(\hat{U}_{1}, \mathscr{L}\right) \stackrel{x_{m} \cdot}{\longrightarrow} \Gamma\left(\hat{U}_{1}, \mathscr{L}\right) \longrightarrow \Gamma\left(\hat{U}_{1}, \mathscr{L} / x_{n} \cdot \mathscr{L}\right) .
$$

If we apply induction to the last module, which is therefore finitely generated over $A_{1} / x_{n} \cdot A_{1} \cong R\left[\left[x_{1}, \ldots, x_{n-1}\right]\right]$, and if we use the fact that $\Gamma\left(\hat{U}_{1}, \mathscr{L}\right)$ is separated and $A_{1}$ is complete in the $x_{n}$-adic topology, we see that $\Gamma\left(\hat{U}_{1}, \mathscr{L}\right)$ is finitely generated over $A_{1}$. It is clear that $\Gamma\left(\hat{U}_{1}, \mathscr{L}\right)$ is torsion-free, and if we localize the exact sequence above in the prime ideal $x_{n} \cdot A_{1} \subseteq A_{1}$ and use induction and Nakayama's lemma, we see that the localiztion of $\Gamma\left(\hat{U}_{1}, \mathscr{L}\right)$ at this prime ideal can be generated by one element over the localization of $A_{1}$, and hence the rank of $\Gamma\left(\hat{U}_{1}, \mathscr{L}\right)$ is $\leqq 1$.

Corollary. If $A, \mathscr{I}, \mathfrak{a}$ are as in Theorem 1 and in addition $A$ is normal, $\Gamma\left(\hat{U}, \mathcal{O}_{0}\right)=A$.

Proof. Any global section of $\mathcal{O}_{0}$ is contained in $Q(A)$. If it were not contained in $A$, its polar divisor would have a nonvoid trace on $\hat{U}$, since its $\operatorname{dimension}$ is $\operatorname{dim}(A)-1$, and so its intersection with $Y$ has $\operatorname{dimension} \geqq \operatorname{dim}(A)$ $-n-1>\operatorname{dim}(A / \mathfrak{a})$ and cannot be contained in $Z=V(A / \mathfrak{a})$.

We now generalize Theorem 1 and solve a problem first posed by A. Ogus ([O], Remark on p. 344).

Theorem 2. Suppose $K$ is a field, $R=K\left[\left[T_{1}, \ldots, T_{n}\right]\right], \mathfrak{p}, \mathfrak{q} \subset R$ prime ideals with $Q(R / \mathfrak{p})$ a regular extension of $K$. Define $A=R / \mathfrak{q}, \mathscr{I}=\mathfrak{p} \cdot A$, and let $\mathfrak{a} \subset A$ be an ideal containing $\mathscr{I} . \quad$ If $\operatorname{dim}(A / \mathscr{I})=\operatorname{dim}(R /(\mathfrak{p}+\mathfrak{q}))=\operatorname{dim}(R / \mathfrak{p})$ $+\operatorname{dim}(R / \mathfrak{q})-n$ (we always have " $\geqq$ " by $[\mathrm{S}], \mathrm{Ch} . \mathrm{V}, \mathrm{Th} .3$ ) and this quantity is $\geqq \operatorname{dim}(A / \mathfrak{a})+2$, then $M(\hat{U})=Q(A)$.

Proof. If $d=\operatorname{dim}(A / \mathfrak{p})$, we may assume that $T_{1}, \ldots, T_{d}$ form a system of parameters for $R / \mathfrak{p}$. We consider the ring $A_{1}=(R / \mathfrak{p}) \hat{\otimes}_{K} A=(R / \mathfrak{p}) \hat{\otimes}_{K}(R / \mathfrak{q})$ and the ideal $\mathscr{I}_{1} \subseteq A_{1}$, which corresponds to the diagonal and is generated by the $T_{i} \widehat{\otimes}_{K} 1-1 \widehat{\bigotimes}_{K} T_{i}$ for $1 \leqq i \leqq n$. Then $A_{1} / \mathscr{I}_{1} \cong R /(\mathfrak{p}+\mathfrak{q}) \cong A / \mathscr{I}$, and $A_{1}$ is an $A$-algebra via the second factor in $(R / \mathfrak{p}) \hat{\otimes}_{K} A$. If we define $\mathfrak{a}_{1}$ as $\mathfrak{a} \cdot A_{1}$, we get a formal scheme $\hat{U}_{1}$ and a bijective flat mapping of formal schemes $\hat{U}_{1} \rightarrow \hat{U}$. Therefore $M(\hat{U}) \subseteq M\left(\hat{U}_{1}\right)$. 
As $A_{1}$ is an integral domain by Lemma 3 and as it fulfills the conditions of Theorem $1, M\left(\hat{U}_{1}\right)=Q\left(A_{1}\right)$, and this is a regular extension of $Q(A)$ by Lemma 3 . Hence it suffices to show that $M(\hat{U})$ is finite over $Q(A)$. For this we consider the minimal prime overideals of $\left(T_{1} \hat{\otimes}_{K} 1-1 \hat{\otimes}_{K} T_{1}, \ldots, T_{d} \hat{\otimes}_{K} 1-1 \hat{\otimes}_{K} T_{d}\right) \subseteq A_{1}$, which we number as $\mathfrak{p}_{2}, \ldots, \mathfrak{p}_{m}$. As $\operatorname{dim}\left(A_{1} / \mathscr{I}_{1}\right)=\operatorname{dim}\left(A_{1}\right)-n$, we get that $\operatorname{dim}\left(A_{1} / \mathfrak{p}_{i}\right)=\operatorname{dim}\left(A_{1}\right)-d=\operatorname{dim}(A)$ for $2 \leqq i \leqq m$, and as

$$
A_{1} /\left(T_{1} \widehat{\otimes}_{K} 1-1 \widehat{\otimes}_{K} T_{1}, \ldots, T_{d} \widehat{\otimes}_{K} 1-1 \widehat{\otimes}_{K} T_{d}\right) \cong(A / \mathfrak{p}) \otimes_{K\left[\left[T_{1}, \ldots, T_{d}\right]\right]} A
$$

is finite over $A$, the $A_{i}$ are finite over $A$, and we must have, that $\mathfrak{p}_{i} \cap A=(0)$ for $2 \leqq i \leqq m$.

If we define $\mathscr{I}_{i}=\mathscr{I}_{1} \cdot A_{i}$ and $\mathfrak{a}_{i}=\mathfrak{a}_{1} \cdot A_{i}$ for $2 \leqq i \leqq m$, we get closed formal subschemes $\hat{U}_{i}$ of $\hat{U}_{1}$, whose set-theoretic union is $\hat{U}_{1}$. All this implies that $M(\hat{U}) \subseteq \bigoplus_{i=2}^{m} M\left(\hat{U}_{i}\right)$. But as the triples $\left(A_{i}, \mathscr{I}_{i}, \mathfrak{a}_{i}\right)$ also fulfill the conditions of Theorem 1 , we have $M\left(\hat{U}_{i}\right)=Q\left(A_{i}\right)$, and therefore $M(\hat{U})$ has finite dimension over $Q(A)$. This proves our theorem.

\section{§3. Algebraization of Formal Subsheaves}

Let $A, \mathscr{I}, \mathfrak{a}, \hat{U}$ be as in the introduction and $M$ a finitely generated $A$ module. $M$ defines a coherent formal sheaf $\mathscr{F}$ on $\hat{U}$, and if $\mathscr{G}$ is a formal subsheaf of $\mathscr{F}$, we may ask whether there exists a submodule $N$ of $M$ such that $\mathscr{G}$ is the formal sheaf associated with $N$. If there exists one such $N$, there exists a canonical one, namely the kernel of the combined mapping $M \rightarrow \Gamma(\hat{U}, \mathscr{F})$ $\rightarrow \Gamma(\hat{U}, \mathscr{F} / \mathscr{G})$.

We call $\mathscr{G}$ algebraic if there is an $N$ as above, and we have the following

Theorem 3. Suppose $A$ is a complete local ring containing a field, $\mathscr{I} \subset A$ an ideal, which can be generated by $n$ elements for some natural number $n$, and for which $A$ is complete in the $\mathscr{I}$-adic topology. Let $\mathfrak{a} \supset \mathscr{I}$ be another ideal with $\mathfrak{a} \neq A$, and let $M$ be a finitely generated $A$-module with associated coherent formal sheaf $\mathscr{F}$ on $\hat{U}$. If $\mathscr{G} \subseteq \mathscr{F}$ is a coherent formal subsheaf, such that for all $f \in \mathfrak{a}$ and all associated prime ideals $\mathfrak{p} \subset A_{(f)}$ of $\Gamma\left(\operatorname{Spf}\left(A_{(f)}\right), \mathscr{F} / \mathscr{G}\right)$

$$
\operatorname{dim}\left(A_{(f)} /\left(\mathfrak{p}+\mathscr{I} \cdot A_{(f)}\right)=\operatorname{dim}\left(A_{(f)} / \mathfrak{p}\right)-n>\operatorname{dim}(A / \mathfrak{a}),\right.
$$

then $\mathscr{G}$ is algebraic.

Remark. It is an easy exercise in commutative algebra, to show, that we only need to test the condition in the theorem for $f$ in some fixed generating 
system of $\mathfrak{a}$, since this implies the condition for all $f$.

Proof of Theorem 3. If we apply Lemma 1 to $\mathscr{F} / \mathscr{G}$ and use the fact that the intersection of algebraic $\mathscr{G}$ 's is algebraic, we see that we may assume that for all $f \in \mathfrak{a}$ and all associated prime ideals $\mathfrak{p}$ of $\Gamma\left(\operatorname{Spf}\left(A_{(f)}\right), \mathscr{F} / \mathscr{G}\right)$ $\operatorname{dim}\left(A_{(f)} / \mathfrak{p}\right)=n+r$ is constant independent from $f$ and $\mathfrak{p}$. As in the proof of the previous theorem we may make a smaller:

This means removing some part of $\hat{U}$. If we have an $N \subseteq M$ which defines $\mathscr{G}$ over the smaller $\hat{U}$, we take the maximal one. Then it is elementary that all associated prime ideals $\mathfrak{p} \subseteq A$ of $M / N$ fulfill $\operatorname{dim}(A / \mathfrak{p})=n+r+1$. Hence for any $f \in \mathfrak{a}$ and all associated prime ideals $\mathfrak{p} \subseteq A_{(f)}$ of $(M / N) \otimes_{A} A_{(f)}$ we also have $\operatorname{dim}\left(A_{(f)} /\left(\mathfrak{p}+\mathscr{I} \cdot A_{(f)}\right)\right) \geqq r>\operatorname{dim}(A / \mathfrak{a})$. But as the submodules $\Gamma\left(\operatorname{Spf}\left(A_{(f)}\right), \mathscr{G}\right)$ and $N \otimes_{A} A_{(f)}$ of $\Gamma\left(\operatorname{Spf}\left(A_{(f)}\right), \mathscr{F}\right)=M \otimes_{A} A_{(f)}$ differ only in the part removed from $\hat{U}$ during the change of $a$, and as the dimension of this part is smaller than the dimension of any associated component of $(M / N) \otimes_{A} A_{(f)}$ or $\Gamma\left(\operatorname{Spf}\left(A_{(f)}\right), \mathscr{F} / \mathscr{G}\right)$, they must be equal.

Let $K \subseteq A$ be a field of representatives and $\mathfrak{b} \subseteq A$ the annihilator of $\Gamma\left(\hat{U},(\mathscr{F} / \mathscr{G}) \otimes_{\mathscr{O}_{0}}\left(\mathcal{O}_{\mathcal{O}} / \mathscr{I} \cdot \mathcal{O}_{\hat{U}}\right)\right)$. Then our conditions imply $\operatorname{dim}(A / \mathfrak{b})=r+1$, and we can chose $x_{1}, \ldots, x_{r+1} \in A$ which form a system of parameters for $(A / \mathfrak{b})$, such that $A / \mathfrak{b}$ is finite over the subring $R=K\left[\left[x_{1}, \ldots, x_{r+1}\right]\right]$ of $A$.

We now replace $\mathfrak{a}$ by $\mathfrak{a}+\mathfrak{b}$ : This enlarges $\hat{U}$, but on the new piece we have a trivial continuation of $\mathscr{G}$, namely by $\mathscr{F}$ itself.

After that we change $\mathfrak{a}$ into $(\mathfrak{a} \cap R) \cdot A+\mathfrak{b}$, which also fulfills the necessary dimension-inequalities.

If $y_{1}, \ldots, y_{n}$ is a system of generators for $\mathscr{I}$, we may form the ring of formal powerseries $A_{1}=R\left[\left[T_{1}, \ldots, T_{n}\right]\right]$, and we get a mapping $A_{1} \rightarrow A$ sending $T_{i}$ to $y_{i}$, which makes $A$ an $A_{1}$-algebra. Let $\mathscr{I}_{1}=\left(T_{1}, \ldots, T_{n}\right) \subset A_{1}$ and define $\mathfrak{a}_{1}$ as the kernel of the combined mapping $A_{1} \rightarrow A \rightarrow A / \mathfrak{a}$, so that $\mathscr{I}=\mathscr{I}_{1} \cdot A$ and $\mathfrak{a}=\mathfrak{a}_{1} \cdot A+\mathfrak{b}$, and therefore we have an adique mapping of formal schemes $\hat{U}-V\left(\mathfrak{a}_{1} \cdot \mathcal{O}_{\hat{U}}\right)$ $\rightarrow \hat{U}_{1}$. If $f \in \mathfrak{a}_{1}$ and $\mathfrak{p} \subset A_{(f)}$ is an associated prime ideal of $\Gamma\left(\operatorname{Spf}\left(A_{(f)}\right), \mathscr{F} / \mathscr{G}\right)$, our construction implies that $A_{(f)} / \mathfrak{p}$ is finite over $A_{1,(f)}$. As both rings have the same dimension and as $A_{1,(f)}$ is integral (it is regular and has no nontrivial idempotents), we have $\mathfrak{p} \cap A_{1 .(f)}=(0)$. Hence we see that $\Gamma\left(\operatorname{Sp} \Gamma\left(A_{(f)}\right), \mathscr{F} / \mathscr{G}\right)$ is finitely generated and torsion-free over $A_{1,(f)}$ and therefore has a well-defined rank s. As $\hat{U}_{1}$ is connected, $s$ is independent of the particular choice of $f \in \mathfrak{a}_{1}$.

Define $N \subseteq M$ as the kernel of the combined mapping $M \rightarrow \Gamma(\hat{U}, \mathscr{F}) \rightarrow$ $\Gamma(\hat{U}, \mathscr{F} / \mathscr{G})$. We want to show that $M / N$ is finite and torsion-free over $A_{1}$ : 
The torsion-freeness is trivial, and for the finiteness it suffices if $A / \operatorname{Ann}(M / N)$ is finite or only integral over $A_{1}$.

So let $x \in A$. If $f \in \mathfrak{a}_{1}$, we look at the characteristic polynomial $F(T)$ of the multiplication with $x$ on the torsion-free $A_{1,(f)}$-module $\Gamma\left(\operatorname{Spf}\left(A_{(f)}\right), \mathscr{F} / \mathscr{G}\right)$. This is a monogeneous polynomial of degree $s$, whose coefficients fit together to global sections of $\mathcal{O}_{\hat{U}_{1}}$. The corollary to Theorem 1 implies $F \in A_{1}[T]$, and according to Hamilton-Cayley the support of $F(x) \cdot(\mathscr{F} / \mathscr{G})$ must be contained in $V\left(\mathfrak{a}_{1} \cdot \mathcal{O}_{0}\right)$ and hence is disjoint from the support of $\mathscr{F} / \mathscr{G}$, which is equal to $V\left(\mathfrak{b} \cdot \mathcal{O}_{\mathfrak{0}}\right)$. Therefore $F(x) \in \operatorname{Ann}(M / N)$.

Let $t$ be the rank of the finite torsion-free $A_{1}$-module $M / N$. As $\mathscr{F} / \mathscr{G}$ is a quotient of the coherent formal sheaf associated with $M / N, s \leqq t$. We can find elements $z_{1}, \ldots, z_{s}$ from $M$ such that for any $f \in \mathfrak{a}_{1}$ the $z_{i}$ form a maximal system of $A_{1,(f)}$-linearly independent elements in $\Gamma\left(\operatorname{Sp} r\left(A_{(f)}\right), \mathscr{F} / \mathscr{G}\right)$. If then $y$ is an arbitrary element of $M$, we can find $c_{0}, \ldots, c_{s}, c_{0} \neq 0$, from $A_{1,(f)}$, such that $c_{0} \cdot y+\sum_{i=1}^{s} c_{i} \cdot z_{i}=0$ in $\Gamma\left(\operatorname{Spf}\left(A_{(f)}\right), \mathscr{F} / \mathscr{G}\right)$. The $c_{i} / c_{0}$ are uniquely determined and therefore glue together to a formal meromorphic function on $\hat{U}_{1}$, which is an element of $Q\left(A_{1}\right)$ according to Theorem 1. Hence we can find such a relation where all the $c_{i}$ lie in $A_{1}$, and then the global section $c_{0} \cdot y+\sum_{i=1}^{s} c_{i} \cdot z_{i}$ of $\mathscr{F} / \mathscr{G}$ vanishes on $V\left(\mathfrak{b} \cdot \mathcal{O}_{0}\right)$, which is the support of $\mathscr{F} / \mathscr{G}$, so that $c_{0} \cdot y+$ $\sum_{i=1}^{s} c_{i} \cdot z_{i} \in N$. This implies $t \leqq s$, so we have $s=t$.

For $f \in \mathfrak{a}_{1}$ the kernel of the canonical surjection $(M / N) \otimes_{A} A_{(f)} \rightarrow$ $\Gamma\left(\operatorname{Spf}\left(A_{(f)}\right), \mathscr{F} / \mathscr{G}\right)$ is now torsion-free of rank $t-s=0$, hence vanishes. So the support of the kernel of the canonical surjection from the formal sheaf associated with $M / N$ to $\mathscr{F} / \mathscr{G}$ is contained in $V\left(\mathfrak{a}_{1} \cdot \mathcal{O}_{0}\right)$, and a simple argument as before shows, that its dimension is too small to allow the kernel not to vanish. This proves our theorem.

We now prove a theorem about the ring of formal powerseries which stands to Theorem 3 in the same relation as Theorem 2 to Theorem 1.

Theorem 4. Suppose $K$ is a field, $\mathscr{I} \subset A=K\left[\left[T_{1}, \ldots, T_{n}\right]\right]$ a prime ideal, such that $Q(A / \mathscr{I})$ is a regular extension of $K, \mathfrak{a} \varsubsetneqq A$ another ideal containing $\mathscr{I}$. Let $M$ be a finitely generated $A$-module and $\mathscr{F}$ its associated coherent sheaf on $\hat{U}$. If $\mathscr{G} \subseteq \mathscr{F}$ is a coherent subsheaf, such that for all $f \in \mathfrak{a}$ and all associated prime ideals $\mathfrak{p} \subset A_{(f)}$ of $\Gamma\left(\operatorname{Spf}\left(A_{(f)}\right), \mathscr{F} / \mathscr{G}\right)$

$$
\operatorname{dim}\left(A_{(f)} /\left(\mathfrak{p}+\mathscr{I} \cdot A_{(f)}\right)\right)=\operatorname{dim}\left(A_{(f)} / \mathfrak{p}\right)-\operatorname{height}_{A}(\mathscr{I})>\operatorname{dim}(A / \mathfrak{a}),
$$


(a priori the first equation is an inequality because of Serre's dimension formula, [S], Ch. V, Th. 3) then $\mathscr{G}$ is algebraic, i.e., there exists a submodule $N$ of $M$ such that $\mathscr{G}$ is the coherent sheaf associated with $N$.

Proof. Let $A_{1}=(A / \mathscr{I}) \hat{\otimes}_{K} A=(A / \mathscr{I})\left[\left[T_{1}, \ldots, T_{n}\right]\right], \mathscr{I}_{1}=\left(T_{1} \hat{\otimes}_{K} 1-1 \hat{\otimes}_{K} T_{1}\right.$, $\left.\ldots, T_{n} \hat{\otimes}_{K} 1-1 \hat{\otimes}_{K} T_{n}\right) \subseteq A_{1}$ and $\mathfrak{a}_{1}=\mathscr{I}_{1}+\mathfrak{a} \cdot A_{1}$, where $A_{1}$ did become an $A$ algebra via the second factor in the complete tensorproduct which defines it. Then we get a formal scheme $\hat{U}_{1}$ with a bijective flat mapping $\hat{U}_{1} \rightarrow \hat{U}$, and as we want to use descent-methods, we also consider $A_{2}=A_{1} \hat{\otimes}_{A} A_{1}=$ $(A / \mathscr{I}) \hat{\otimes}_{K}(A / \mathscr{I}) \hat{\otimes}_{K} A$ with its ideal

$$
\begin{aligned}
& \mathscr{I}_{2}=\left(T_{1} \hat{\otimes}_{K} 1 \hat{\otimes}_{K} 1-1 \hat{\otimes}_{K} T_{1} \hat{\otimes}_{K} 1, \ldots, T_{n} \hat{\otimes}_{K} 1 \hat{\otimes}_{K} 1-1 \hat{\otimes}_{K} T_{n} \hat{\otimes}_{K} 1,\right. \\
& \left.1 \hat{\otimes}_{K} T_{1} \hat{\otimes}_{K} 1-1 \hat{\otimes}_{K} 1 \hat{\otimes}_{K} T_{1}, \ldots, 1 \hat{\otimes}_{K} T_{n} \hat{\otimes}_{K} 1-1 \hat{\otimes}_{K} 1 \hat{\otimes}_{K} T_{n}\right)
\end{aligned}
$$

and with $\mathfrak{a}_{2}=\mathscr{I}_{2}+\mathfrak{a} \cdot A_{2}$, where $A_{2}$ becomes an $A$-algebra via the second complete tensorproduct in its definition. The first tensorproduct induces two $A$-linear mappings $j_{1}, j_{2}: A_{1} \rightarrow A_{2}$, given by $j_{1}(a)=a \widehat{\bigotimes}_{A} 1$ and $j_{2}(a)=1 \widehat{\bigotimes}_{A} a$. So we get a diagram of flat mappings

$$
A \longrightarrow A_{1} \underset{j_{2}}{\stackrel{j_{1}}{\longrightarrow}} A_{2}
$$

where all the mappings respect the different $\mathscr{I}$-adic topologies, and so we have a diagram of formal schemes:

$$
\hat{U} \longleftarrow \hat{U}_{1} \underset{j_{2}}{\stackrel{j_{1}}{\leftrightarrows}} \hat{U}_{2} .
$$

We define $\mathscr{F}_{i}$ and $\mathscr{G}_{i}$ for $i=1,2$ as the pullbacks of $\mathscr{F}$ resp. $\mathscr{G}$ on $\hat{U}_{i}$. Then the $\mathscr{F}_{i}$ can be defined by the finitely generated $A_{i}$-modules $M_{i}=M \otimes_{A} A_{i}$.

We intend to show first that $\mathscr{F}_{1}$ can be defined by a submodule $N_{1} \subseteq M_{1}$. If we have any such submodule, we get submodules $N_{1} \otimes_{A_{1} j_{1}} A_{2}$ and $N_{1} \otimes_{A_{1} j_{2}} A_{2}$ of $M_{2}$. If these are equal there exists a submodule $N \subseteq M$, such that $N_{1}$ is the submodule $N \otimes_{A} A_{1}$ of $M_{1}=M \otimes_{A} A_{1}$ : If we would use the usual tensorproduct instead of the complete tensorproduct this would be an immediate consequence of the usual descent-theory. But we can reduce to this case:

If $\mathrm{m}$ is the maximal ideal of $A$, we first replace in the definition of $A_{1}$ and $A_{2} A / \mathscr{I}$ by $A /\left(\mathscr{I}+\mathfrak{m}^{h}\right)$ for some natural number $h$. Then the complete tensorproduct in the definition becomes an ordinary one, and if we apply descenttheory to the submodule induced by $N$ on $M \otimes_{A}$ (new $A_{1}$ ), we obtain an unique $N \subseteq M$ inducing our $N_{1}$. By uniqueness this $N$ is independent of $h$, and it is 
easy to see that $N \otimes_{A} A_{1}=N_{1}$. To show that $\mathscr{F}_{1}$ is algebraic we use Theorem 3. To fulfill the conditions of this theorem comes down to proving that the fibers of $\hat{U}_{1} \rightarrow \hat{U}$ have no imbedded primes. Hence we only need the following

Lemma 4. If $f \in \mathfrak{a}$, then the fibers of the mapping $A_{(f)} \rightarrow A_{1,(f)}$ have no imbedded primes, i.e., they fulfill the $S_{1}$-condition.

Proof of Lemma 4. We choose $x_{1}, \ldots, x_{d} \in A$ which form a system of parameters for $A / \mathscr{I}$. Then $A / \mathscr{I}$ is finite over its subring $K\left[\left[x_{1}, \ldots, x_{d}\right]\right]$, and $A_{1}=(A / \mathscr{I}) \hat{\bigotimes}_{K} A \cong A\left[\left[x_{1}, \ldots, x_{d}\right]\right] \otimes_{K\left[\left[x_{1}, \ldots, x_{d}\right]\right]}(A / \mathscr{I}) . \quad$ If $\mathfrak{p} \subseteq A_{(f)}$ is a prime ideal, $A_{1,(f)} / \mathfrak{p} \cdot A_{1,(f)}$ is the $\mathscr{I}_{1}$-adic completion of $(\mathscr{I}$-adic completion of $\left.A\left[\left[x_{1}, \ldots, x_{d}\right]\right]_{f}\right) \otimes_{A_{(f)}}\left(A_{(f)} / \mathfrak{p}\right) \otimes_{K\left[\left[x_{1}, \ldots, x_{d}\right]\right]}(A / \mathscr{I})$.

As all our rings have dualizing complexes and as therefore their formal fibers are gorenstein, we need only to show that the latter ring has no imbedded primes, and as this ring is a submodule of the direct sum of a finite number of copies of its subring $\left(\mathscr{I}\right.$-adic completion of $\left.A\left[\left[x_{1}, \ldots, x_{d}\right]\right]_{f}\right) \otimes_{A_{(f)}}\left(A_{(f)} / \mathfrak{p}\right)$ (since $A / \mathscr{I}$ can be imbedded in the direct sum of a finite number of copies of its subring $\left.K\left[\left[x_{1}, \ldots, x_{d}\right]\right]\right)$, we only have to consider this subring. But as the rings $A_{(f)}$ and ( $\mathscr{I}$-adic completion of $A\left[\left[x_{1}, \ldots, x_{d}\right]\right]_{f}$ ) are regular, any flat mapping between them has fibers which are complete intersections, and this remark finishes the proof of our lemma.

Now we know that $\mathscr{G}_{1}$ is algebraic, and hence is induced by $N_{1}=$ kernel of the combined mapping $M \rightarrow \Gamma\left(\hat{U}_{1}, \mathscr{F}_{1}\right) \rightarrow \Gamma\left(\hat{U}_{1}, \mathscr{F}_{1} / \mathscr{G}_{1}\right)$. We want to show that the submodules $N_{1} \otimes_{A_{1} j_{1}} A_{2}$ and $N_{1} \otimes_{A_{1} j_{2}} A_{2}$ of $M_{2}$ are both equal to the kernel of the combined mapping $M_{2} \rightarrow \Gamma\left(\hat{U}_{2}, \mathscr{F}_{2}\right) \rightarrow \Gamma\left(\hat{U}_{2}, \mathscr{F}_{2} / \mathscr{G}_{2}\right)$ or equivalently that the mapping $\left(M_{1} / N_{1}\right) \otimes_{A_{1} j_{1}} A_{2} \rightarrow \Gamma\left(\hat{U}_{2}, \mathscr{F}_{2} / \mathscr{G}_{2}\right)$ is injective (and the same for $j_{2}$ instead of $j_{1}$ ). Lemma 3 immediately implies that for any prime ideal $\mathfrak{p} \subset A_{1} j_{1}(\mathfrak{p}) \cdot A_{2}$ is also a prime ideal, and by the usual calculation the associated primes of $\left(M_{1} / N_{1}\right) \otimes_{A_{1} j_{1}} A_{2}$ are therefore of the form $j_{1}(\mathfrak{p}) \cdot A_{2}$ with some associated prime $\mathfrak{p} \subseteq A_{1}$ of $M_{1} / N_{1}$. If such a prime $j_{1}(\mathfrak{p}) \cdot A_{2}$ is associated to the kernel of our mapping $\left(M_{1} / N_{1}\right) \otimes_{A_{1} j_{1}} A_{2} \rightarrow \Gamma\left(\hat{U}_{2}, \mathscr{F}_{2} / \mathscr{G}_{2}\right)$, we must have $j_{1}(\mathfrak{p}) \cdot \mathcal{O}_{\mathrm{O}_{2}}=\mathcal{O}_{\mathrm{O}_{2}}$ and hence by faithfully flat descent $\mathfrak{p} \cdot \mathcal{O}_{\hat{U}_{1}}=\mathcal{O}_{\hat{U}_{1}}$, which is impossible, since $\mathfrak{p}$ is associated to $M_{1} / N_{1}$, which is contained in $\Gamma\left(\hat{U}_{1}, \mathscr{F}_{1} / \mathscr{G}_{1}\right)$.

After all we now know that there is a submodule $N \subseteq M$ for which $N \otimes_{A} A_{1}$ $=N_{1}$. The pullback of the coherent subsheaf of $\mathscr{F}$ defined by $N$ to $\hat{U}_{1}$ is equal to the pullback of $\mathscr{G}$, and by faithfully flat descents $\mathscr{G}$ is the algebraic subsheaf of $\mathscr{F}$ which is defined by $N$. This proves our theorem. 


\section{§4. Descent-Methods}

We now consider rings $A$ which are not necessarily complete in the topology defined by the maximal ideal but only in the $\mathscr{I}$-adic topology. By the previous theorems our formal objects become algebraic after the base-change to the completion in the topology defined by the maximal ideal, and we want to use descent to prove that our original objects are algebraic.

Theorem 5. Suppose $A$ is a local ring, $\mathscr{I} \subset \mathfrak{a} \varsubsetneqq A$ ideals, such that $A$ is complete in the $\mathscr{I}$-adic topology. Assume further that $A$ contains a field and has a dualizing complex. If either

i) $\mathscr{I}$ is generated by $n$ elements for some natural number $n$, or

ii) $A$ is regular, $\mathscr{I}$ a prime ideal of height $n$, such that the completion of $A / \mathscr{I}$ is integral and such that $\hat{A}$, the completion of $A$, contains a field of representatives $K$ for which $Q(\hat{A} / \mathscr{I} \cdot \hat{A})$ is a regular extension of $K$, we have the following results, where $\hat{U}$ etc. are defined as in the introduction:

a) If $\mathfrak{p} \subset A$ is a prime ideal, such that for $A_{1}=A / \mathfrak{p}, \mathscr{I}_{1}=\mathscr{I} \cdot A_{1}, \mathfrak{a}_{1}$ $=\mathfrak{a} \cdot A_{1}, \operatorname{dim}\left(A_{1} / \mathscr{I}_{1}\right)=\operatorname{dim}\left(A_{1}\right)-n \geqq \operatorname{dim}\left(A_{1} / \mathfrak{a}_{1}\right)+2$ (we always have “ $\geqq$ " in the first equality), then $M\left(\hat{U}_{1}\right)=Q\left(A_{1}\right)$.

b) If $M$ is a finitely generated A-module with associated sheaf $\mathscr{F}$ on $\hat{U}$, and if $\mathscr{G} \subseteq \mathscr{F}$ is a coherent formal subsheaf such that for all $f \in \mathfrak{a}$ and all associated prime ideals $\mathfrak{q} \subset A_{(f)}$ of $\Gamma\left(\operatorname{Spf}\left(A_{(f)}\right), \mathscr{F} / \mathscr{G}\right)$ $\operatorname{dim}\left(A_{(f)} /\left(\mathfrak{q}+\mathscr{I} \cdot A_{(f)}\right)\right)=\operatorname{dim}\left(A_{(f)} / \mathfrak{q}\right)-n \geqq \operatorname{dim}(A / \mathfrak{a})+2$ (we again have always " $\geqq$ " in the first equality), then there exists a submodule $N \subseteq M$ such that $\mathscr{G}$ can be defined by $N$.

Proof. a) is an easy consequence of b), if we consider the graph of a formal meromorphic function. To prove b) we make the base-change to $A_{1}=\hat{A}$ $=$ completion of $A$ with its ideals $\mathscr{I}_{1}=\mathscr{I} \cdot A_{1}$ and $\mathfrak{a}_{1}=\mathfrak{a} \cdot A_{1}$, the finitely generated $A_{1}$-module $M_{1}=M \otimes_{A} A_{1}$ and the formal sheaves $\mathscr{F}_{1}$ and $\mathscr{G}_{1}$ on $\hat{U}_{1}$, which of course are the pullbacks of $\mathscr{F}$ and $\mathscr{G}$. The previous two theorems imply that $\mathscr{G}_{1}$ can be defined by a submodule $N_{1} \subseteq M_{1}$, for which we take the kernel of the combined mapping $M \rightarrow \Gamma\left(\hat{U}_{1}, \mathscr{F}_{1}\right) \rightarrow \Gamma\left(\hat{U}_{1}, \mathscr{F}_{1} / \mathscr{G}_{1}\right)$. For any natural number $d$ the sheaf $\mathscr{G}+\mathscr{I}^{d} \cdot \mathscr{F} \subseteq \mathscr{F}$ is algebraic and can be defined by $N_{d} \subseteq M$, which is the kernel of the combined mapping 


$$
M \rightarrow \Gamma(\hat{U}, \mathscr{F}) \rightarrow \Gamma\left(\hat{U}, \mathscr{F} /\left(\mathscr{G}+\mathscr{I}^{d} \cdot \mathscr{F}\right)\right)
$$

We are ready if we show that for any natural number $m$ the decreasing sequence of the submodules $N_{d}+\mathscr{I}^{m} \cdot M$ (for $d \rightarrow \infty$ ) of $M$ becomes stationary:

For if $L_{m}$ is its limit, we have for $k \geqq m, L_{m}=L_{k}+\mathscr{I}^{m} \cdot M$, and the $\mathscr{I}$-adic completeness of $M$ implies that the submodule $L=\bigcap_{m \in N} L_{m}=\varliminf_{(m}\left(L_{m} / \mathscr{I}^{m} \cdot M\right) \subseteq M$ fulfills $L_{m}=L+\mathscr{I}^{m} \cdot M$ and that therefore $\mathscr{G}$ can be defined by $L$.

If we make the base-change from $A$ to $A_{1}, N_{d}$ changes into its counterpart on $A_{1}$, and by faithfully flat descent we only have to prove that the descending sequence of these counterparts becomes stationary. So we may assume that $A$ is complete. We also may replace $M_{1}$ by $M_{1} / N_{1}$, and then we see that for all associated primes $\mathfrak{p} \subseteq A$ of $M_{1}$ height $_{\left(A_{1} / \mathfrak{p}\right)}\left(\mathfrak{a}_{1} \cdot A_{1} / \mathfrak{p}\right)>n$, since otherwise we would get a contradiction to our assumption about the associated prime ideals of the $\Gamma\left(\operatorname{Spf}\left(A_{1,(f)}\right), \mathscr{F}_{1} / \mathscr{G}_{1}\right)$. From now on we drop the subscripts for $A_{1}$ and assume that $A=A_{1}$.

If we write $A$ as the quotient of some big regular local ring it is easy to derive case i) from ii), so that we only have to consider the latter. Our $N_{d}$ are now given by $N_{d}=\cup_{k \in N}\left(\mathscr{J}^{d} \cdot M: \mathfrak{a}^{k}\right)$, and so it is cnough to prove the following

Lemma 5. Suppose $A$ is a regular local ring, $\mathscr{I} \subseteq A$ a prime ideal of height $n, \mathfrak{a} \subseteq A$ another ideal containing $\mathscr{I}$. If $M$ is a finitely generated A-module, such that for all associated prime ideals $\mathfrak{p}$ of $M$ height $_{(A / \mathfrak{p})}(\mathfrak{a} \cdot A / \mathfrak{p})>n$, then for all $d \in \mathbb{N}$ there exists an $m \geqq d$ with $\underset{k \in \mathbb{N}}{\cup}\left(\mathscr{I}^{m} \cdot M: \mathfrak{a}^{k}\right) \subseteq \mathscr{I}^{d} \cdot M$.

Proof. We start with the remark that the conditions for our lemma remain true if we complete $A$ in the $\mathscr{I}$-adic topology or if we localize in a prime ideal $\mathfrak{q} \supseteq \mathscr{I}$ of $A$. We use induction over $\operatorname{dim}(A / \mathfrak{a})$. If $\operatorname{dim}(A / \mathfrak{a})=0$, the modules of the decreasing sequence $\left(\cup_{k \in \mathcal{N}}\left(\mathscr{I}^{m} \cdot M: \mathfrak{a}^{k}\right)+\mathscr{I}^{d} \cdot M\right) / \mathscr{I}^{d} \cdot M(m \rightarrow \infty)$ have finite length and hence become stationary, so that for big $m$ they are equal to $L_{d} / \mathscr{I}^{d} \cdot M$ for some submodule $L_{d} \subseteq M$.

For $s \leqq t$ we have $L_{s}=L_{t}+\mathscr{I}^{s} \cdot M$, and hence if some $L_{d}$ is not equal to $\mathscr{I}^{d} \cdot M$ we get that $\bigcap_{d \in N} L_{d}=\varliminf_{i m}\left(L^{d} / \mathscr{I}^{d} \cdot M\right) \subseteq \lim \left(M / \mathscr{I}^{d} \cdot M\right)$ is different from zero. (We may assume that $A$ is complete in the $\mathscr{I}$-adic topology.) We may choose an element $x \in M$ which is contained in all $L_{d}$ and for which $A \cdot x \cong A / p$ for some associated prime ideal $\mathfrak{p}$ of $M$. The $\mathscr{I}$-adic and the a-adic topology on $M$ then induce the same topology on $A \cdot x$, which by Artin-Rees in the $\mathscr{T}$-adic resp. a-adic topology of $A \cdot x \cong A / p$. But this is impossible, since by Scrre's 
dimension-inequality ([S], Ch. V, Th. 3)

$$
\operatorname{height}_{(A / \mathfrak{p})}(\mathscr{I} \cdot A / \mathfrak{p}) \leqq \operatorname{height}_{A}(\mathscr{I})=n<\operatorname{height}_{(A / \mathfrak{p})}(\mathfrak{a} \cdot A / \mathfrak{p}) .
$$

Therefore the $L_{d}$ are equal to $\mathscr{I}^{d} \cdot M$, and this implies our lemma in the case $\operatorname{dim}(A / \mathfrak{a})=0$.

If $\operatorname{dim}(A / \mathfrak{a})>0$, we consider for fixed $d$ the modules

$$
\left(\cup_{k \in N}\left(\mathscr{I}^{m} \cdot M: \mathfrak{a}^{k}\right)+\mathscr{I}^{d} \cdot M\right) / \mathscr{I}^{d} \cdot M
$$

for $m \rightarrow \infty$. As the supports of these modules decrease with increasing $m$, they become constant, and as the localizations of these modules in any prime ideal of $A$ different from $\mathrm{m}$, the maximal ideal, tend to zero by induction, they have for big $m$ finite length.

For a fixed $d$ we can by the first case find an $l \geqq d$ with $\underset{k \in N}{\cup}\left(\mathscr{I}^{l} \cdot M: \mathrm{m}^{k}\right)$ $\subseteq \mathscr{I}^{d} \cdot M$, and by the preceding considerations there exists an $m \geqq l$ with

$$
\bigcup_{k \in N}\left(\mathscr{I}^{m} \cdot M: \mathfrak{a}^{k}\right) \subseteq \underset{k \in N}{\bigcup}\left(\mathscr{I}^{l} \cdot M: \mathfrak{m}^{k}\right)
$$

Hence we have $\underset{k \in N}{\cup}\left(\mathscr{I}^{m} \cdot M: \mathfrak{a}^{k}\right) \subseteq \mathscr{I}^{d} \cdot M$, which is exactly what we had to prove.

\section{$\S 5$. Connectedness-Theorems}

We now start with the applications of our theory. As in the classical case the algebraization of formal functions implies results about connectedness.

Theorem 6. Let $A$ be a local ring containing a field which has a dualizing complex, $\mathscr{I} \subset \mathfrak{a} \subset A$ two ideals, such that $\mathscr{I}$ can be generated by $n$ elements for some natural number $n$. If $A$ is an integral domain and is $\mathscr{I}$-adically complete and if $\operatorname{dim}(A / \mathfrak{a}) \leqq \operatorname{dim}(A)-n-2$, then $\operatorname{Spec}(A / \mathscr{I})-\operatorname{Spec}(A / \mathfrak{a})$ is connected.

Proof. We use induction over $n$. If $n=1$, we may assume that $\mathscr{I} \neq 0$, and then we may apply case a) of Theorem 5:

As $M(\hat{U})$ is a field, $\hat{U}$ must be connected. But this is exactly what we have to prove. For general $n$ we choose an element $x \in \mathscr{I}$ such that $\mathscr{I} \mid A \cdot x$ can be generated by $n-1$ elements. If $\mathfrak{p}_{1}, \ldots, \mathfrak{p}_{h}$ are the minimal prime overideals of $A \cdot x$, the case $n=1$ implies that, if we allow repetitions in the sequence $\mathfrak{p}_{1}, \ldots, \mathfrak{p}_{h}$, we may assume that $\operatorname{dim}\left(A /\left(\mathfrak{p}_{i}+\mathfrak{p}_{i+1}\right)\right) \geqq \operatorname{dim}(A)-2$ for $1 \leqq i \leqq h-1$, so that 
$\operatorname{dim}\left(A /\left(\mathfrak{p}_{i}+\mathfrak{p}_{i+1}+\mathscr{I}\right)\right)>\operatorname{dim}(A / \mathfrak{a})$. As by induction the

$$
\operatorname{Spec}\left(A /\left(\mathscr{I}+\mathfrak{p}_{i}\right)\right)-\operatorname{Spec}\left(A /\left(\mathfrak{a}+\mathfrak{p}_{i}\right)\right)
$$

are connected and as by reasons of dimension $\operatorname{Spec}\left(A /\left(\mathscr{I}+\mathfrak{p}_{i}+\mathfrak{p}_{i+1}\right)\right)$ is not contained in $\operatorname{Spec}(A / \mathfrak{a})$, we may conclude that $\operatorname{Spec}(A / \mathscr{I})-\operatorname{Spec}(A / \mathfrak{a})$ must be connected.

Corollary. Suppose $K$ is a field, $\mathfrak{p}, \mathfrak{q} \subset A=K\left[\left[T_{1}, \ldots, T_{n}\right]\right]$ prime ideals, such that $Q(A / \mathfrak{p})$ is a regular extension of $K$. If $\mathfrak{a} \subset A$ is another ideal which contains $\mathfrak{p}+\mathfrak{q}$ and for which

$$
\operatorname{dim}(A / \mathfrak{a}) \leqq \operatorname{dim}(A / \mathfrak{p})+\operatorname{dim}(A / \mathfrak{q})-n-2,
$$

then $\operatorname{Spec}(A /(\mathfrak{p}+\mathfrak{q}))-\operatorname{Spec}(A / \mathfrak{a})$ is connected.

Proof. By Lemma $3(A / \mathfrak{p}) \widehat{\otimes}_{K}(A / \mathfrak{q})$ is an integral domain, and $A /(\mathfrak{p}+\mathfrak{q})$ is isomorphic to the quotient of this ring after the ideal generated by the $T_{i} \hat{\otimes}_{K} 1-1 \hat{\otimes}_{K} T_{i}$ for $1 \leqq i \leqq n$.

We also can prove a generalization of Zariski's connectedness-theorem:

Theorem 7. Suppose $K$ is a field, $X, Y, K$-schemes of finite type which are integral and such that $Y$ is normal, $f: X \rightarrow Y$ a proper surjective mapping such that $f_{*}\left(\mathcal{O}_{X}\right)=\mathcal{O}_{Y}$ or equivalently such that the field of meromorphic functions of $Y$, which via $f$ becomes a subfield of the field of meromorphic functions of $X$, is algebraically closed in this field. If then $Z \subset X$ is a closed subscheme with $\operatorname{dim}(Z) \leqq \operatorname{dim}(X)-\operatorname{dim}(Y)-2$, the fibers of the induced mapping $X-Z$ $\rightarrow Y$ are connected.

Proof. We may replace $X$ by its normalization and $Z$ by its inverse image in this normalization and therefore we may assume that $X$ is normal. As the fibers of $f: X \rightarrow Y$ are connected by Zariski's connectedness-theorem (which by the way can be derived from Theorem 6), we only have to prove that for any closed point $x \in Z$ the intersection of $\operatorname{Spec}\left(\mathcal{O}_{X, x}\right)-Z$ with the fiber of $f$ over $x$ is connected. We call the completion of $\mathcal{O}_{X, x}$, which is again normal and therefore integral, $A$, and the ideal in $A$ which defines the preimage of $Z$ is named $a$. If then $y=f(x)$ and if $t_{1}, \ldots, t_{d} \in \mathcal{O}_{Y, y}$ form a system of parameters $(d=\operatorname{dim}(Y))$, Theorem 6 implies that $\operatorname{Spec}\left(A /\left(t_{1}, \ldots, t_{d}\right)\right)-\operatorname{Spec}\left(A /\left(\mathfrak{a}+\left(t_{1}, \ldots, t_{d}\right)\right)\right)$ is connected, and so is its image in $X$, which is exactly the set of points whose connectedness we have to prove. 
We also get the main result of $[\mathrm{FH}]$ :

Theorem 8. Suppose $K$ is a field, $P=\mathbb{P}_{K}^{m} \times \boldsymbol{P}_{K}^{m} \times \cdots \times \mathbb{P}_{K}^{m}$ the product of $r$ copies of the projective $m$-space, and $\Delta \subseteq P$ the image of the diagonal embedding of $\mathbb{P}_{K}^{m}$ in $P$. If $X$ is an irreducible $K$-variety and $f: X \rightarrow P$ a proper mapping with $\operatorname{dim}(f(X))>(r-1) \cdot m$, then $f^{-1}(\Delta)$ is connected.

Proof. By using Zariski's connectedness-theorem we may assume that $f$ is finite. Consider the product of the projective cones over the $\mathbb{P}_{K}^{m}$ and the completion of its local ring in the product of the vertices. This is a complete regular local ring, and the closed subset of its spectrum corresponding to the preimage of $\Delta$ can be defined by $(r-1) \cdot(m+1)$ equations. $X$ corresponds to a finite integral algebra over this ring of dimension $\geqq(r-1) \cdot(m+1)+2$, and if we remove from its spectrum the maximal ideal and intersect with the preimage of $\Delta$, we obtain something connected according to Theorem 6 . From this it is easy to derive our theorem.

\section{§6. Formal Subschemes of the Projective Space}

If we apply our results to the completion of the local ring in the vertex of the projective cone over the projective space, we immediately obtain the following results:

Theorem 9. Let $K$ be a field, $X, Y \subseteq \boldsymbol{P}_{K}^{n}$ irreducible subvarieties such that $X$ is geometrically integral. If $\operatorname{dim}(X \cap Y)=\operatorname{dim}(X)+\operatorname{dim}(Y)-n(X$ and $Y$ intersect properly), and if $Z \subseteq X \cap Y$ is a closed subscheme with $\operatorname{dim}(Z)$ $\leqq \operatorname{dim}(X \cap Y)-2($ where $\operatorname{dim}(\varnothing)=-1)$, then $(X \cap Y)-Z$ is $G 3$ in $Y-Z$.

Theorem 10. Let $K$ be a field, $X \subseteq \mathbb{P}_{K}^{n}$ a geometrically integral subscheme defined by the ideal $\mathscr{J}(X) \subset \mathcal{O}_{P_{K}^{n}}$. Let $Z \subseteq X$ be a closed subscheme and $U=\mathbb{P}_{K}^{n}-Z$. Define $\hat{U}$ as the formal completion of $U$ along $U \cap X$. If $\mathscr{F}$ is a coherent formal sheaf on $\hat{U}$ which is induced from an algebraic sheaf on $\boldsymbol{P}_{K}^{n}$ and if $\mathscr{G} \subseteq \mathscr{F}$ is a formal subsheaf such that for all $x \in \hat{U}$ and all associated prime ideals $\mathfrak{p} \subset \mathcal{O}_{0, x}$ of $(\mathscr{F} / \mathscr{G})_{x}, \quad \operatorname{dim}\left(\mathcal{O}_{0, x} /\left(\mathfrak{p}+\mathscr{J}(X) \cdot \mathcal{O}_{0, x}\right)\right)=$ $\operatorname{dim}\left(\mathcal{O}_{\hat{0}, x} / \mathfrak{p}\right)-n+\operatorname{dim}(X) \geqq \operatorname{dim}(Z)+2$ (where again $\operatorname{dim}(\varnothing)=-1$ ). Then $\mathscr{G}$ also can be defined by an algebraic sheaf on the $\boldsymbol{P}_{K}^{n}$, which furthermore can be taken as a subsheaf of the algebraic sheaf defining $\mathscr{F}$.

We also may apply our theory to complex-analytic geometry: If we 
complete the complex projective space along a closed subvariety, any analytic object in a neighbourhood of our subvariety induces a formal complex-analytic object. By the usual GAGA-theory this object is formal algebraic, and we may apply our theory to conclude that sometimes this object is algebraic in the usual sense. In this way we obtain the following results:

Theorem $\mathbb{1 1}$. If $X$ and $Y$ are irreducible closed subvarieties of the $\mathbb{P}_{\boldsymbol{C}}^{n}$ which intersect properly and whose intersection is not discrete, any meromorphic function defined on a connected neighbourhood (in the metric topology) of $X \cap Y$ in $Y$ can be extended to the whole of $Y$.

Theorem 12. If $X$ is an irreducible subvariety of the $\mathbb{P}_{c}^{n}$ defined by the ideal $\mathscr{J}(X) \subset \mathcal{O}_{\mathbb{P}_{C}^{n}}$, if $\mathscr{F}$ is a coherent analytic sheaf on $\mathbb{P}_{C}^{n}$ and if $\mathscr{G} \subseteq \mathscr{F}$ is a coherent analytic subsheaf defined in a neighbourhood of $X$ in $\mathbb{P}_{\mathbb{C}}^{n}$ (in the metric topology), such that for all $x \in X$ and all associated prime ideals $\mathfrak{p} \subset \mathcal{O}_{\mathbb{P}_{C}^{n}, x}$ of $(\mathscr{F} / \mathscr{G})_{X} \operatorname{dim}\left(\mathcal{O}_{\mathbb{P}_{C}^{n}, x} /\left(\mathfrak{p}+\mathscr{J}(X) \cdot \mathcal{O}_{\mathbb{P}_{C}^{n}, x}\right)\right)=\operatorname{dim}\left(\mathcal{O}_{\mathbb{P}_{C}^{n}, x} / \mathfrak{p}\right)-n+\operatorname{dim}(X)$ $>0$, then there exists a coherent subsheaf of $\mathscr{F}$ on the whole $\mathbb{P}_{C}^{n}$ which coincides with $\mathscr{G}$ in a smaller neighbourhood of $X$.

\section{References}

[ F ] Faltings, G., Zur Existenz dualisierender Komplexe, Math. Zeitschrift, 162 (1978), 75-86.

[FH] Fulton, W. and Hansen, J., A connectedness theorem for projective varieties, with applications to intersections and singularities of mappings, Ann. of Maih., 109 (1979), 159-166.

[ H ] Hartshorne, R., Amplc Subvarieties of Algebraic varieties, Lecture Notes in Math., 156, Springer, 1970.

[HM] Hironaka, H. and Matsumura, H., Formal functions and formal embeddings, $J$. Math. Soc. Japan, 20 (1968), 52-82.

[ M ] Matsumura, H., Commutative Algebra, Benjamin, New York, 1970.

[ N ] Nagata, M., Local Rings, Interscience, New York, 1962.

[ O ] Ogus, A. Local cohomological dimension of algebraic varieties, Ann. of Math., 98 (1973), 327-365.

[ S ] Serre, J.-P., Algèbre Locale Multiplicités, Lecture Notes in Math., 11 Springer, 1965. 
